bioRxiv preprint doi: https://doi.org/10.1101/640185; this version posted May 21, 2019. The copyright holder for this preprint (which was not certified by peer review) is the author/funder. All rights reserved. No reuse allowed without permission.

Levetiracetam inhibits SV2A-synaptotagmin interaction

\title{
Levetiracetam inhibits SV2A-synaptotagmin interaction at synapses that lack SV2B
}

Kristine Ciruelas, Daniele Marcotulli, Jane M Sullivan, Sandra M Bajjalieh*

Departments of Pharmacology and \&Pysiology and Biophysics

University of Washington

Seattle, WA

* To whom correspondence should be addressed at bajjalie@uw.edu

\begin{abstract}
Epilepsy remains a difficult-to-treat neurological disorder prompting the need for new therapies that work via alternate mechanisms. Levetiracetam (LEV) is the first in a series of anti-epilepsy drugs that target presynaptic functioning. LEV binds the synaptic vesicle protein SV2A, and has been shown to decrease neurotransmitter release in hippocampal slices. The molecular basis of LEV action is unknown, however, and direct effects of LEV on SV2A function remain to be determined. SV2A is the most widely expressed paralog of a three-gene family (SV2A, B, C) that is variably co-expressed throughout the CNS. All three SV2s bind the calcium sensor protein synaptotagmin and SV2 plays a crucial role in synaptotagmin stability and trafficking. Here we addressed the action of LEV at the cellular and molecular level asking whether the presence of non-LEV binding SV2 paralogs influences drug action and whether LEV impacts SV2A's role in synaptotagmin function. We report that LEV altered short-term synaptic plasticity in isolated neurons from SV2B knockout but not wild-type mice, mimicking the loss of SV2 function. Similarly, LEV reduced SV2A binding to synaptotagmin only in the absence of SV2B. Furthermore, LEV reduced and slowed the internalization of synaptotagmin in neurons cultured from SV2B KO but not WT mice. Taken together, these findings suggest that LEV alters synaptic release probability by disrupting SV2's regulation of synaptotagmin selectively in neurons that express only SV2A. Neurons that meet this requirement include most inhibitory neurons and the granule cells of the dentate gyrus, two classes of neuron implicated in epilepsy.
\end{abstract}

\section{Introduction}

Levetiracetam (Keppra ${ }^{\text {TM }}$, LEV) is a second-generation anti-epileptic drug (AED) that lacks many of the adverse side effects of classic AEDs. In addition to its use for epilepsy, LEV is being used to treat a range of neurological disorders and has recently been found to be a promising treatment for cognitive impairment (1). Hindering its further development is the fact that LEV's molecular mechanism of action remains unknown.

Consistent with its unique pharmacological profile, LEV has a novel target, Synaptic Vesicle protein 2A, (SV2A) (2). SV2A is one of three functionally redundant membrane proteins unique to secretory vesicles that undergo calcium-regulated secretion (3). SV2A is both necessary and sufficient for LEV binding (4), and multiple observations suggest that LEV acts by affecting SV2A function. This includes the fact that SV2A hypomorphic $\left(\mathrm{SV} 2 \mathrm{~A}^{+/-}\right)$mice are less responsive to LEV's anti-seizure effects (5). Furthermore, the ability of structurally-related compounds to control seizures is directly correlated to affinity for SV2A (6). Although SV2 has been proposed to have multiple functions $(7,8)$, its only fully vetted action is modulating the expression levels and trafficking of the calcium sensor protein synaptotagmin via a calcium- and phosphorylation-regulated interaction between the two proteins (9-11).

Of the three SV2 genes, SV2A is the most widely expressed. Notably, it is the only SV2 expressed in the majority of GABAergic neurons and dentate granule cells of the hippocampus $(12,13)$, two classes of neurons implicated in the control of excitability. Loss of 
bioRxiv preprint doi: https://doi.org/10.1101/640185; this version posted May 21, 2019. The copyright holder for this preprint (which was not certified by peer review) is the author/funder. All rights reserved. No reuse allowed without permission.

Levetiracetam inhibits SV2A-synaptotagmin interaction

SV2A, but not other SV2 paralogs, results in severe seizures and early death $(14,15)$. Interestingly, elevated SV2A is also associated with pathological neurotransmission. Seizure kindling in rodents is correlated with increased SV2A expression (16-18), and overexpression of SV2A in cultured neurons results in aberrant neurotransmission that is rescued by LEV (19). Thus, it appears that both decreased and increased SV2A may contribute to epilepsy.

LEV produces an activity-dependent decrease of both excitatory and inhibitory neurotransmission in hippocampal slice preparations $(20,21)$. However, it has no effect on isolated, hippocampal neurons (19), suggesting that LEV action might not be via a direct effect on SV2A. Alternatively, because most neurons express both SV2A and SV2B, the lack of effect the presence of non-LEV binding SV2 paralogs may mask LEV's effects. To begin to understand LEV's mechanism of action at the cellular level, we examined LEV effects in neurons in which SV2A was the sole SV2 paralog. We report that in preparations from SV2B knockout (BKO) mice, LEV reduces synaptic depression in autaptic neurons, reduces SV2A binding to synaptotagmin, and slows synaptotagmin endocytosis in conventionally cultured hippocampal neurons. Our findings suggest that LEV acts primarily at synapses that express only SV2A by disrupting SV2Aregulated synaptotagmin function.

\section{Results}

LEV decreases synaptic depression in hippocampal neurons from BKO mice

Previous studies examining LEV effects on synaptic transmission in autaptic hippocampal neurons found no measureable effect on neurotransmission (19). The wild-type (WT) excitatory neurons used in those studies express SV2A and SV2B with low or nonexistent levels of SV2C. Because all SV2 paralogs can rescue normal neurotransmission when expressed in neurons from SV2 ${ }^{-/-} \mathrm{SV} 2 \mathrm{~B}^{-}$ ${ }^{1-}$ mice (DKO) mice (7), they are considered to be functionally redundant. Thus, the presence of SV2B, which does not bind LEV, might be masking measureable effects in WT excitatory neurons.

To assess LEV effects on neurotransmission in the absence of SV2B, we measured synaptic depression, an indicator of synaptic release probability (22). Excitatory principal neurons from the $\mathrm{CA}$ region of the hippocampus were cultured from SV2B ${ }^{-1-}$ (BKO) mice. Single neurons were grown on microislands of astrocytes allowing them to form autaptic synapses. Cultures were treated +/- 300uM LEV for 3-7 hours. This concentration and incubation time was chosen because it produced effects on synaptic transmission in hippocampal slices (23). Excitatory postsynaptic currents (EPSCs) in response to $2 \mathrm{sec}, 20 \mathrm{~Hz}$ stimulus trains were recorded.

Cultured excitatory hippocampal neurons normally demonstrate synaptic depression during $20 \mathrm{~Hz}$ stimulus trains. LEV treatment reduced the extent of this depression in neurons from BKO mice (Figure 1A). Consistent with this, the paired-pulse ratio, a comparison of the first two EPSCs, was increased by LEV treatment $\left(\mathrm{PPR}_{\mathrm{Ctrl}}=\right.$ $\left.0.87+0.06, \quad P R_{L E V}=1.02+0.04,{ }^{*} P<0.05\right)$ (Figure 1B). These changes in the rate of synaptic depression suggest that LEV is decreasing the probability of neurotransmitter release from BKO synapses (24). We note that this effect is similar to the decrease in synaptic depression observed in neurons lacking SV2 (25), suggesting that LEV impairs SV2 function. In contrast to its effects on neurons lacking SV2B, LEV had no significant effect on synaptic depression in neurons cultured from WT mice $\left(\mathrm{PPR}_{\mathrm{ctrl}}=0.82+0.05, \mathrm{PPR}_{\mathrm{LEV}}=\right.$ $0.94+0.05$ ) (Figure 1C-D). The selective effect of LEV on transmitter release from neurons expressing only SV2A suggests that the presence of other SV2 paralogs masks LEV effects in WT hippocampal neurons.

LEV decreases SV2A binding to synaptotagmin

SV2 binds the C2B domain of the calcium sensor protein synaptotagmin (10,26-29), domain essential to synaptotagmin's role in exo (30-32) and endocytosis $(33,34)$. To determine if LEV affects the interaction of 


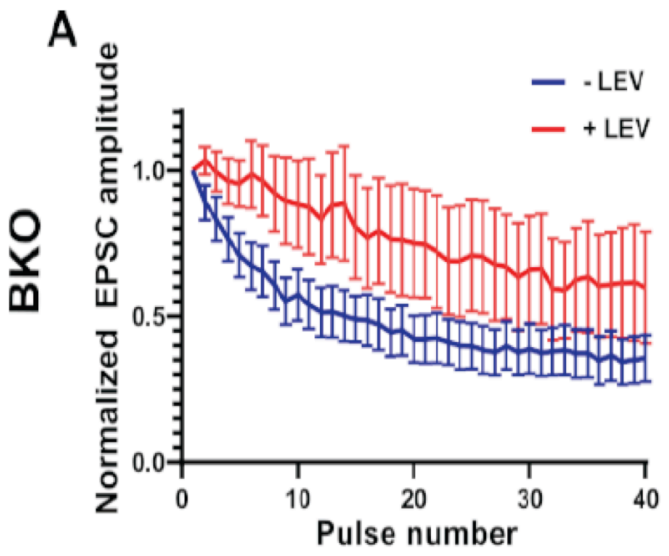

B

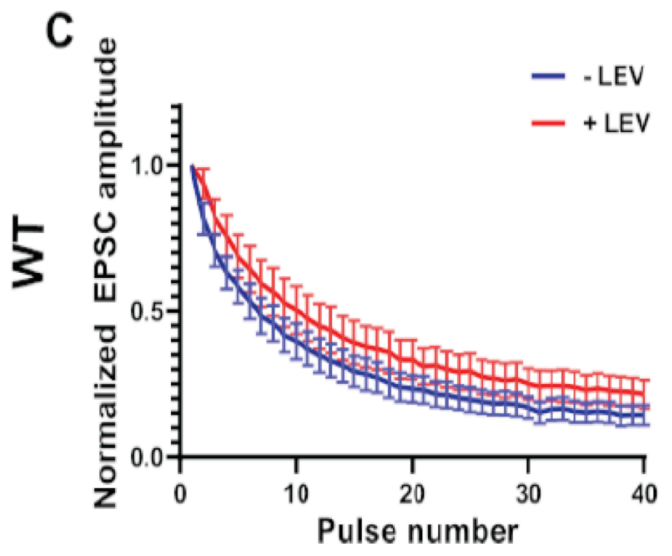

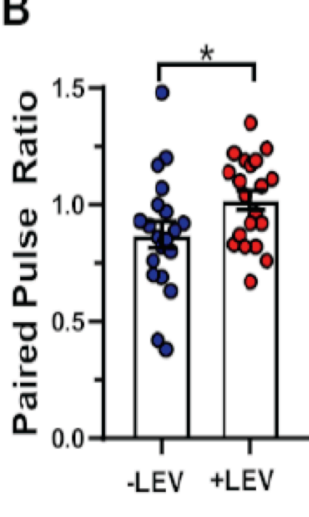

D

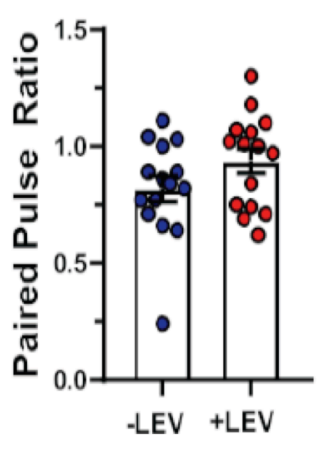

Figure 1: LEV decreases synaptic depression, an indicator of release probability, in isolated hippocampal neurons from SV2 BKO mice.

Excitatory postsynaptic potentials (EPSCs) were measured using whole cell patch clamp stimulation and recording from isolated (autaptic) pyramidal neurons cultured on microislands of astrocytes.

(A,C) Shown are averaged EPSC amplitudes in response to a $2 \mathrm{~s}, 20 \mathrm{~Hz}$ train. Amplitudes were normalized to the initial response then averaged. (A) BKO and (C) WT neurons treated +/- 300uM LEV for 3-7 hours. LEV decreased synaptic depression only in BKO neurons, suggesting that it reduces synaptic release probability in synapses that express only SV2A.

(B,C) Paired pulse ratio, an index of synaptic release probability, was calculated as $\mathrm{EPSC}_{2} / \mathrm{EPSC}_{1}$. LEV (+LEV) increased the paired pulse ratio in neurons from BKO mice $\left(P R_{+L E V}=1.02 \pm 0.04\right.$, student's t-test, ${ }^{\star} P<0.05, \mathrm{n}=20$ ) compared to control (-LEV) $\left(P P R_{c t r l}=0.87 \pm 0.06, n=20\right)$. In contrast, LEV had no effect on the paired pulse ratio in neurons from WT $\left(P R_{+L E V}=0.94 \pm 0.05\right.$, $\left.\mathrm{n}=15, \mathrm{PPR}_{\text {ctrl }}=0.82 \pm 0.05, \mathrm{n}=15\right)$. Data represent the mean \pm SEM.
SV2A with synaptotagmin, we assessed their binding in vitro in the presence and absence of 300uM LEV. Binding reactions included Triton $\mathrm{X}-100$ extracts of mouse brain and resin coated with either recombinant glutathione-Stransferase (GST) or a glutathione-Stransferase-synaptotagmin 1 fusion protein (GST-Syt1)(27). Because calcium inhibits SV2 - synaptotagmin binding, we assayed LEV action in both low calcium $\left(10^{-9} \mathrm{M}\right)$ and high $(2 \mathrm{mM})$ calcium. SV2A binding to the resin was then assessed by immunoblot analysis.

LEV treatment reduced SV2A binding to GSTSyt1 in both high and low calcium conditions when the source of SV2A was brain extracts of BKO mice. At low calcium concentrations, LEV reduced binding by $\sim 40 \%$. Calcium alone decreased binding by $63 \%$, and addition of LEV resulted in a further decrease to $14 \%$ of control (86\% reduction) (Figure 2A). Somewhat surprisingly, LEV had no effect on
SV2A binding to synaptotagmin when the source of SV2A was WT mouse brain (Figure 2B). The absence of LEV effects on SV2A when SV2B is present suggests that SV2s may form hetero-oligomers, precluding LEV action. Consistent with this, we find that SV2B coimmunoprecipitates with SV2A from extracts of WT mouse brain (data not shown).

LEV slows synaptotagmin endocytosis at synapses

The interaction of SV2A and synaptotagmin plays a crucial role in synaptotagmin's ability to engage the proteins that mediate clathrin-dependent endocytosis (35). Thus SV2A is essential to synaptotagmin trafficking to synaptic vesicles $(9,11)$. To determine if LEV's inhibition of SV2Asynaptotagmin binding impacts synaptotagmin trafficking, we assessed its effects using two measures of synaptotagmin endocytosis in cultured hippocampal neurons. 

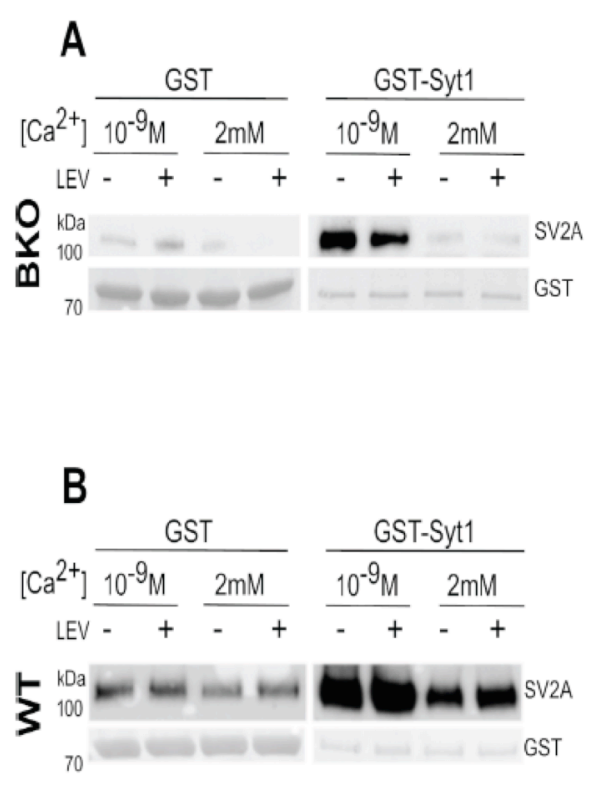
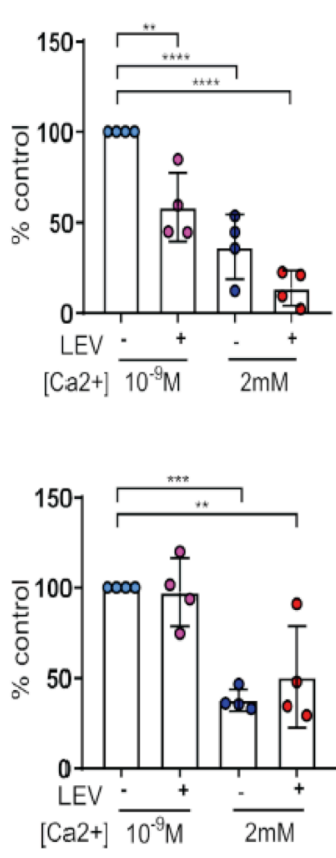

Figure 2: LEV decreases SV2A binding to synaptotagmin in vitro in the absence of SV2B. LEV effects of the SV2A-synaptotagmin interaction were assessed using a GST pull-down approach. The fusion protein GST- synaptotagmin 1 (GSTSyt1), or GST as a control, was attached to glutathione resin and incubated with extracts of mouse brain in the presence or absence of 300uM LEV and in low $\left(10^{-9} \mathrm{M}\right)$ or high $(2 \mathrm{mM})$ calcium. SV2A binding was assessed by immunoblot analysis and normalized to the amount of GST-Syt1 or GST in the same lane. Binding was graphed as the percentage of the no LEV, low calcium (Control) condition. (A) SV2A from extracts of BKO mouse brain shows reduced binding to GST-Syt1 in the presence of LEV. Consistent with previous studies, high calcium reduced binding, and addition of LEV in the presence of high calcium further reduced binding (n=4, One-way ANOVA ** $P<0.01$, *** $P<0.0001$ ). Blots from a representative experiment are shown at the left. (B) SV2A from extracts of WT mouse brain showed reduced binding to GST-Syt1 in the presence of high calcium. In contrast to extracts from BKO mice, LEV had no effect on the interaction $\left(\mathrm{n}=4\right.$, One-way ANOVA ${ }^{* \star} P<0.01,{ }^{* \star \star} P<0.0001,{ }^{\star \star \star \star *}$ $P<0.0001$ ). Blots from a representative experiment are shown at the left. Bar graphs represent data \pm SD.
First, we quantified the amount of surface stranded synaptotagmin in conventional hippocampal cultures treated with LEV. Cultures from WT or BKO mice were treated +/- 300uM LEV for 3 hours and then incubated with EZ-Link Sulfo-NHS-LC-LC-Biotin, a membrane-impermeant biotinylating reagent. After cultures were harvested and extracted in Triton X-100, surface stranded biotinylated proteins were isolated using streptavidin beads. The amount of biotinylated synaptotagmin and SV2A was quantified using Western blot analysis. Blots were probed for SV2A, synaptotagmin, and the plasma membrane protein sodium/potassium ATPase, which served as a loading control. Compared to untreated cells, LEV treatment increased the amount of synaptotagmin on the surface by $\sim 60 \%$, suggesting decreased internalization (Figure 3A). Interestingly, there was no significant effect on the amount of biotinylated SV2A suggesting that SV2A's internalization is not affected by LEV. Consistent with its effects on neurotransmission and in vitro protein interaction, LEV had no effect on the amount of biotinylated synaptotagmin or SV2A in WT neurons (Figure 3B). To control for the possibility that increased surface synaptotagmin was due to increased expression levels, we compared the amount of synaptotagmin in brain post nuclear supernatant from WT and BKO mice. There was no difference (data not shown). Thus, the most parsimonious interpretation is that LEV disrupts SV2A regulation of synaptotagmin trafficking.

To measure LEV effects on the rate of synaptotagmin retrieval, we tracked the exoand endocytosis of synaptotagmin1-pHluorin (Syt1-pHluorin), a fusion protein consisting of synaptotagmin 1 and pHluorin, a $\mathrm{pH}$ sensitive GFP variant (36). When fused to the lumenal domain of a synaptic vesicle protein, pHluorin is quenched by the low $\mathrm{pH}$ of the vesicle interior. It becomes fluorescent upon vesicle fusion and exposure to the neutral $\mathrm{pH}$ of the synapse exterior. As pHluorin fusion proteins are recycled back into vesicles during endocytosis, fluorescence is quenched. The rate of fluorescence decline can be used to measure the endocytotic time constant, tau. 

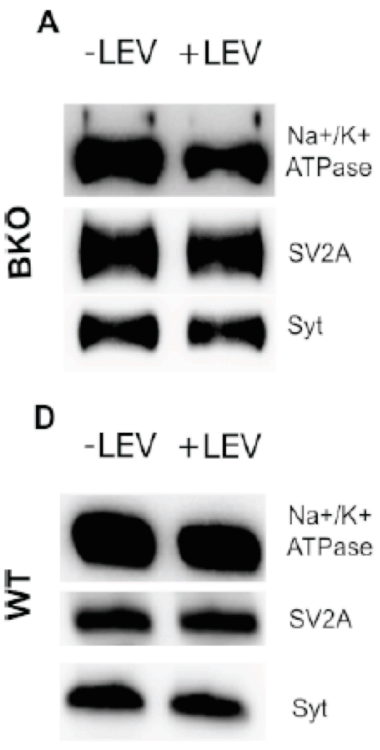

C
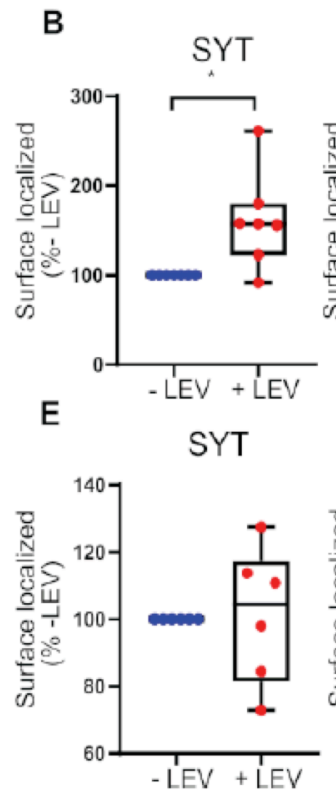

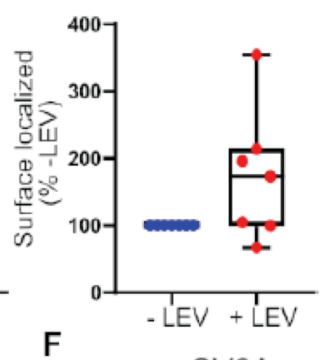

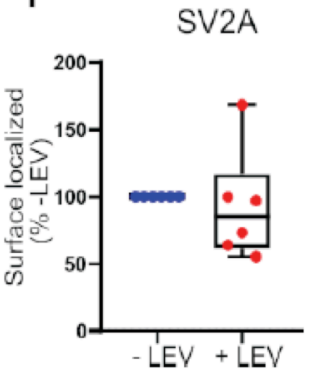

Figure 3: LEV increases surface localization of synaptotagmin in hippocampal neurons cultured from SV2 BKO mice.

To assess LEV's effect on SV2's role in synaptotagmin trafficking, we compared the amount of surface localized synaptotagmin in hippocampal neurons treated +/-300uM LEV for three hours. Shown are representative immunoblots (A, D) and quantification of biotinylated synaptotagmin (Syt1) (B, E) and SV2A (C, F) in BKO and WT hippocampal neurons. To control for protein loading, the amount of biotinylated Syt1 or SV2A was normalized to biotinylated sodium potassium ATPase $(\mathrm{Na}+/ \mathrm{K}+$ ATPase). Values were expressed as the percentage of the no LEV condition. (B) LEV increased the amount of Syt1 on the surface in neurons from BKO mice (Mann-Whitney, ${ }^{*} P<0.05$, $n=7$ ) (C) LEV did not affect the amount of surfacelocalized SV2A. In neurons from WT mice, LEV had no significant effect on the amount of either Syt1 (E) or SV2A (F) in WT neurons $(n=5)$. Each data point represents a different culture (n).

Conventional hippocampal cultures from BKO mice were transfected with a construct driving the expression of Syt1-pHluorin under the control of the synapsin promoter. Prior to field stimulation, cultures were treated +/300uM LEV for 3-8 hours after which fluorescence was recorded at a rate of $2 \mathrm{~Hz}$ in response to a stimulus train of 200 action potentials delivered at $40 \mathrm{~Hz}$. LEV treatment slowed Syt1-pHluorin retrieval after the initial stimulus train. LEV increased the endocytic time constant by $\sim 60 \%\left(\tau_{\text {ctrl }}=53.9+5.4 \mathrm{~s}, \tau_{\text {Lev }}=\right.$ $86.7 \pm 10.2 \mathrm{~s}$ ) (Figure 4A-C). In contrast, LEV treatment had no significant effect on the time course of Syt1-pHluorin retrieval in cultures from WT mice $\left(\tau_{\text {ctrl }}=49.69+5.18 \mathrm{~s}, \tau_{\mathrm{LEV}}=\right.$ $55.6+7.44 \mathrm{~s}$ ), consistent with it affecting only synapses that exclusively express SV2A (Figure 4 D-E).

Because complete loss of SV2A results in early death, it has been impossible to test the requirement for SV2A in LEV action. But because the cultures used for these studies are generated from neonatal mice (Postnatal days 0-2), we were able to compare LEV action in cultures from BKO from DKO mice. Consistent with a requirement for SV2A, LEV had no effect on the time course of Syt1-
pHluorin retrieval in cultures from DKO mice $\left(\tau_{\text {ctrl }}=60.2+7.55 \mathrm{~s}, \tau_{\mathrm{Lev}}=52.13+3.34 \mathrm{~s}\right.$ ) (Figure 4F-H).

Loss of SV2A leads to a selective effect on the trafficking of synaptotagmin. To assess the specificity of the LEV action on endocytosis, we measured retrieval of synaptophysin, a vesicle protein whose trafficking is not linked to SV2 $(9,11)$. For these studies, we tracked the exo- and endocytosis of synaptophysin-pHluorin (SypHy) in cultures from both WT and BKO mice. In contrast to LEV's effects on Syt1-pHluorin trafficking, LEV had no significant effect on SypHy retrieval in either BKO $\left(\tau_{\mathrm{ctrl}}=61.17 \pm 6.4 \mathrm{~s}, \quad \tau_{\mathrm{LEV}}=\right.$ $56.5+3.81 \mathrm{~s})$ or WT $\left(\tau_{\mathrm{ctrl}}=55.45+6.92 \mathrm{~s}, \tau_{\mathrm{LEV}}=\right.$ $45.92+3.92$ s) cultures (Figure 5). This suggests that LEV has a selective effect on proteins that bind to SV2A.

\section{Discussion}

The work presented here provides two insights into the mechanism LEV action. First, we show that LEV action is affected by the presence of other SV2 paralogs. suggesting that LEV action predominates at synapses that express SV2A exclusively. This finding has important implications not only for 

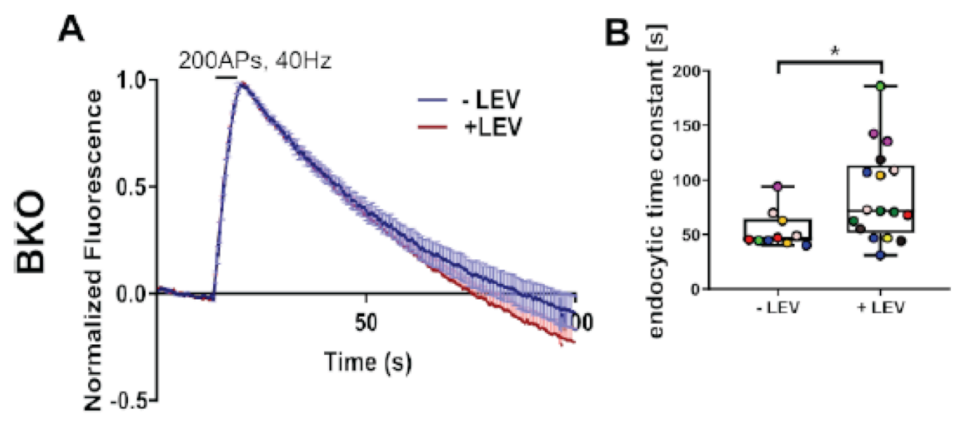

\section{Figure 4: LEV slows Syt1-pHluorin retrieval in hippocampal neurons from SV2 BKO mice}

To assess the effects of LEV on synaptotagmin trafficking, which is regulated by SV2A, we measured the fluorescence decay of Syt1-pHluorin in neurons from BKO, WT and DKO mice
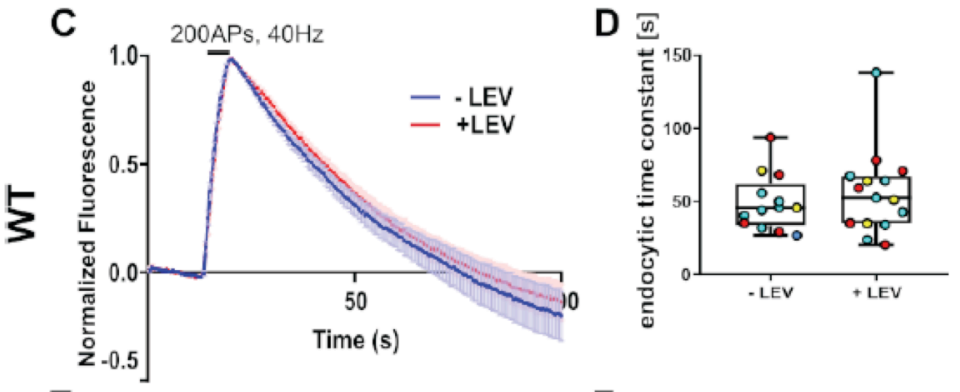
cultured on glass coverslips. Average time course $(A, C, E)$ and endocytic time constants (B, D, F) of Syt1-pHluorin fluorescence in response to 200APs, delivered at $40 \mathrm{~Hz}$ are shown.

(A, B) In neurons from BKO mice, LEV increased the endocytic time constant $(t)$ indicating slower endocytosis of Syt1-
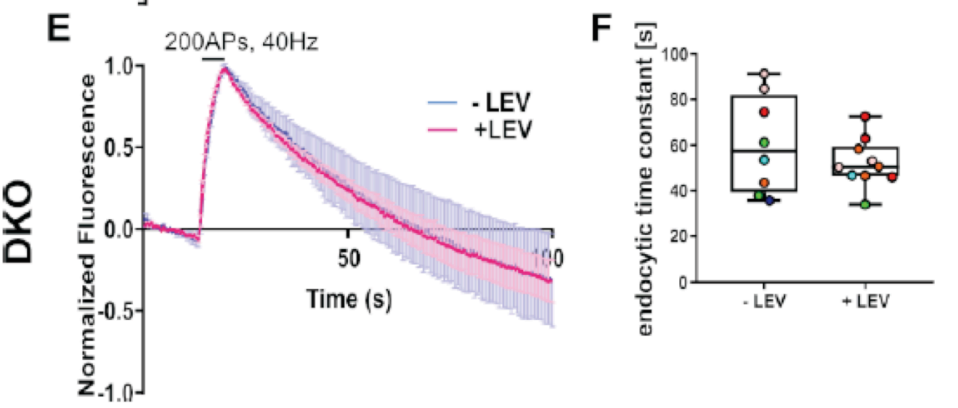
pHluorin $\left(\tau_{\text {ctrl }}=53.9 \pm 5.4 \mathrm{~s}, \mathrm{n}=6, \tau_{\text {Lev }}=\right.$ $86.7 \pm 10.2$ s, $n=9$, Mann-Whitney, ${ }^{*} P$ $<0.05)$.

(C, D) LEV had no effect on Syt1-pHluorin endocytosis in in WT neurons $\left(\tau_{\text {ctrl }}=\right.$ $49.69 \pm 5.18 \mathrm{~s}, \mathrm{n}=4, \tau_{\mathrm{LEV}}=55.6 \pm 7.44 \mathrm{~s}$, $\mathrm{n}=3$ ).

understanding how LEV reduces seizure activity, but also for the development of compounds that target other SV2 paralogs. Second, we demonstrate that LEV acts at the molecular level by disrupting SV2A's interaction with the calcium sensor protein synaptotagmin. These findings provide further evidence that LEV action is unique among antiepileptic medications.

LEV action predominates at synapses that express only SV2A

A standing puzzle in the search for LEV's cellular action has been the absence of an effect on isolated neurons (19), which precluded genetic or structure-function studies of LEV action at the cellular level. Our finding that LEV reduced synaptic depression in isolated hippocampal neurons from BKO but not WT mice allows us to re-evaluate previous hypotheses of LEV action.
Selectivity for synapses that contain only SV2A suggests that LEV acts narrowly in the brain, despite the widespread expression of SV2A. SV2A is co-expressed with SV2B in most brain synapses. The exceptions are the majority of GABAergic synapses and the excitatory projections of dentate granule neurons in the hippocampus, which contain only SV2A $(12,13)$. Both types of synapses have been implicated in the control of brain excitability $(37,38)$. The targeted action of LEV at synapses that play a major role in the development and control of seizures may be the basis for its efficacy and high therapeutic index. Furthermore, because SV2A expression has been reported to increase in some forms of epilepsy $(18,39)$, determining whether LEV has more widespread action in epileptic brain will be important to refining its therapeutic use.

Because our preparation utilizes neurons before the early death induced by loss 


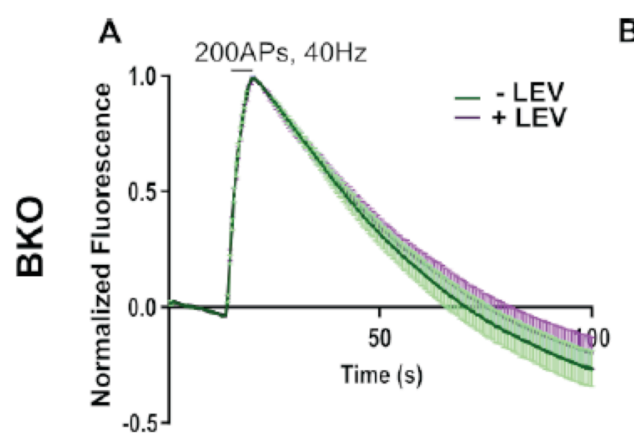

B
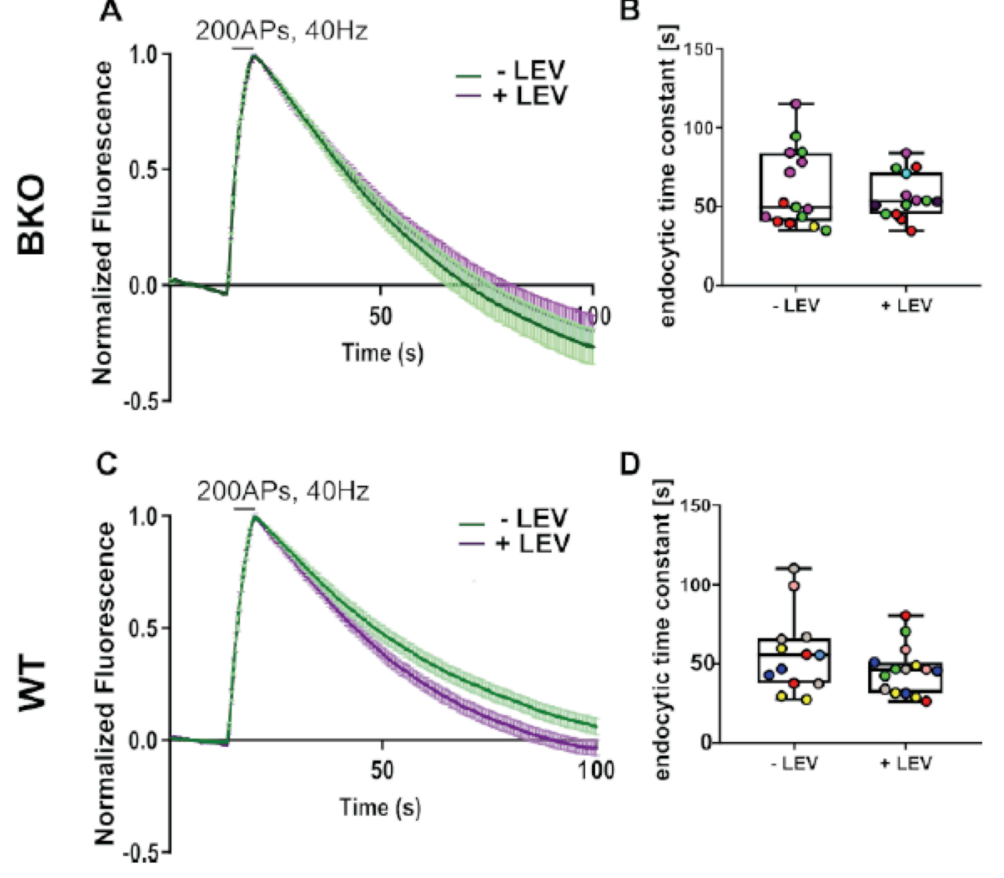

\begin{abstract}
Figure 5: LEV does not affect SypHy retrieval in WT of BKO hippocampal neurons.

To assess the effects of LEV on synaptophysin trafficking, which is not regulated by SV2A, we measured the fluorescence decay of synaptophysin-pHluorin (SypHy) in neurons from BKO, WT and DKO mice cultured on glass coverslips. Average time course (A, C) and endocytic time constants (B, D) of SypHy fluorescence in response to 200APs, delivered at $40 \mathrm{~Hz}$ are shown. Average time course $(A, C)$ and endocytic time constants $(B, D)$ of synaptophysin (SypHy) in BKO (A,B) and WT (C,D) hippocampal neurons in response to 200APs, $40 \mathrm{~Hz}$. LEV did not affect SypHy endocytosis in BKO neurons $\left(\tau_{c t r l}=\right.$ $61.17 \pm 6.4 \mathrm{~s}, \mathrm{n}=4, \tau_{\mathrm{LEV}}=56.5 \pm 3.81 \mathrm{~s}, \mathrm{n}=5$ ) or in WT neurons $\left(\tau_{\mathrm{ctrl}}=55.45 \pm 6.92 \mathrm{~s}, \mathrm{n}=2, \quad \tau_{\mathrm{LEV}}=\right.$ $45.92 \pm 3.92 \mathrm{~s}, \mathrm{n}=3$ ). Average time course are graphed as mean \pm SEM. Endocytic time constants are graphed using box plots. Culture dates $(n)$ are indicated by color. Each data point represents a separate coverslip.
\end{abstract}

of SV2A, we were able to rigorously test the requirement for SV2A in LEV action. The absence of a LEV effect in neurons from DKO mice is the first demonstration that loss of SV2A abolishes LEV action.

\section{LEV inhibits SV2A binding to synaptotagmin}

Although multiple functions have been ascribed to SV2 (8,40-42), it has one verified function. SV2 binds to and regulates the stability and trafficking of synaptotagmin $(9,11,26,29,43)$. The fact that synaptotagmin expression levels and trafficking are disrupted with loss of SV2 suggests that SV2 plays an escort or chaperone-like function. Because synaptotagmin plays an essential role in both exo- and endocytosis, it suggests that LEV's ultimate mechanism of action may be the disruption of synaptotagmin function. This interpretation is consistent with the previous finding that LEV restores normal synaptotagmin levels in synapses overexpressing SV2A (19). It also supports the conclusion that regulation of synaptotagmin is a primary function of SV2 (44). Thus, LEVmediated inhibition of the SV2A-synaptotagmin interaction could have a direct effect on synaptotagmin's ability to engage with the vesicle fusion (SNARE) complex (32).
Synaptotagmin plays an essential role in regulating vesicle exocytosis by interacting with membrane lipids and the proteins that mediate vesicle fusion (SNAREs) (32). By disrupting SV2A's chaperone or escort action, LEV could affect synaptotagmin's ability to contribute to exocytosis. Consistent with this, we note that loss of SV2, which LEV treatment recapitulated in our studies of synaptic plasticity, reduces synaptic vesicle priming and results in fewer assembled vesicle fusion (SNARE) complexes $(25,45)$.

Synaptotagmin has also been implicated in endocytosis (34). SV2A promotes the binding of synaptotagmin to AP2, a component of the clathrin-dependent endocytosis machinery (35). Our observation that LEV slows synaptotagmin endocytosis and decreases its internalization suggests that by disrupting the SV2A-synaptotagmin interaction, LEV's mechanism of action includes affecting synaptotagmin retrieval. This suggests that LEV could act by decreasing the amount of synaptotagmin in recycling vesicles. The interpretation that LEV reduces the replenishment of release competent vesicles is consistent with previous studies reporting that LEV produces an activity-dependent decrease 
bioRxiv preprint doi: https://doi.org/10.1101/640185; this version posted May 21, 2019. The copyright holder for this preprint (which was not certified by peer review) is the author/funder. All rights reserved. No reuse allowed without permission.

Levetiracetam inhibits SV2A-synaptotagmin interaction

in synaptic transmission (20) via faster supply rate depression (46). In contrast, a LEV effect on synaptotagmin recycling or function suggests an alternate explanation for the observation that LEV requires exocytosis in order to be effective. Rather than requiring vesicle exocytosis to get LEV into synapses (21), our finding that LEV disrupts SV2A binding to synaptotagmin suggests that LEV action is limited to vesicles that have yet to be formed and/or primed for release.

In summary, our results provide initial insights into the molecular action of LEV. Further analysis of how LEV impacts protein interactions in presynaptic terminals will be the next stage towards developing a structural approach to further drug development.

\section{Experimental Procedures}

Animals and reagents

The generation of $\mathrm{SV} 2 \mathrm{~A}^{+/+} \mathrm{SV} 2 \mathrm{~B}^{-/-}$(BKO) and $S V 2 A^{-1-} S V 2 B^{-/-}(D K O)$ mice was reported previously (25). Wild-type (WT) and BKO mice were kept as separate colonies generated from littermate heterozygous crosses. All animals were on a $99.99 \% \quad \mathrm{C} 57 \mathrm{BL} / 6$ genetic background. DKO mice were generated by crossing SV2A ${ }^{+/} S V 2 B^{-/-}$mice. Genotype was determined by PCR before culturing neurons. The animal protocol was reviewed and approved by the Institutional Animal Care and Use Committee of the University of Washington.

Levetiracetam (LEV) was from SigmaAldrich (L8668-100MG). Polyclonal anti-SV2A antibody was house generated and affinity purified from sera (13), rabbit polyclonal antisynaptotagmin antibody was raised against the cytoplasmic domain, and anti-Na+/K+ ATPase was from Development Studies Hybridoma Bank (a6F). Antibody binding in immunoblot analyses was detected using horse radish peroxidase-conjugated secondary antibodies and visualized using enhanced chemiluminescent chemistry (BioRad Clarity ${ }^{\mathrm{TM}}$ ) and visualized via Bio-Rad Chemidoc. Immunoreactive bands were quantified using ImageLab software (BioRad). Secondary antibodies were used at a 1: 5000 dilution (Invitrogen HRP-conjugated goat anti-rabbit G-
21234 and HRP-conjugated goat anti-mouse G-21040).

\section{Tissue Culture/Transfections}

For electrophysiological recordings, neurons isolated from the $\mathrm{CA}$ region of the hippocampus of P0-P1 mice were cultured on small microislands of permissive substrate as previously described (25). Neurons were plated on a feeder layer of astrocytes, then grown in neural medium without mitotic inhibitors and used for recordings after 12-16 days in vitro (DIV). For all other experiments, CA hippocampal neurons were cultured on $25 \mathrm{~mm}$ coverslips coated with poly-D-lysine and rat collagen and layer of astrocytes as previously described (9).

Cultures were transfected using calcium phosphate at 6-8 DIV. Synaptotagmin1pHluorin (Syt1-pHluorin) was obtained from Dr. Volker Haucke (Leibniz Institute of Molecular Pharmacology). Synaptophysin-pHluorin (SypHy) was obtained from Dr. Leon Lagnado (University of Sussex). For transfections, cultures were serum starved for 1.5 hours prior to transfection. A calcium phosphate/DNA precipitate was formed in HEPES-buffered saline $[280 \mathrm{mM} \mathrm{NaCl}, 10 \mathrm{mM} \mathrm{KCl}, 1.5 \mathrm{mM}$ $\mathrm{NaH}_{2} \mathrm{PO}_{4}, 12 \mathrm{mM}$ D-glucose, 50mM HEPES, $\mathrm{pH}$ 7.08] for 20-25 minutes. The precipitate (200uL) was added drop-wise to $2 \mathrm{~mL}$ serum free media and incubated for $1.5 \mathrm{hrs}$. After transfection, the cultures were returned to their original culture medium.

\section{Electrophysiology}

Following treatment +/- 300 uM LEV for 3$7 \mathrm{hrs}$, whole-cell voltage clamp recordings were made from excitatory neurons on singleneuron islands using an Axopatch 200A amplifier (Axon Instruments, Sunnyvale, CA). The extracellular recording solution contained $119 \mathrm{mM} \mathrm{NaCl}, 5 \mathrm{mM} \mathrm{KCl}, 2 \mathrm{mM} \mathrm{CaCl}, 1.5 \mathrm{mM}$ $\mathrm{MgCl}_{2}, 30 \mathrm{mM}$ glucose, $20 \mathrm{mM}$ HEPES, and 1 uM glycine; 300 uM LEV or water (vehicle) was added to the external solution of cells during recording. The pipette solution contained 148.5 $\mathrm{mM}$ K-gluconate, $9 \mathrm{mM} \mathrm{NaCl}, 1 \mathrm{mM} \mathrm{MgCl} 2,10$ mM HEPES, and $0.2 \mathrm{mM}$ EGTA. Cells were held at $-60 \mathrm{mV}$ and stimulated with a $1 \mathrm{~ms}$ depolarization to $+20 \mathrm{mV}$ to evoke neurotransmitter release. Recording 
bioRxiv preprint doi: https://doi.org/10.1101/640185; this version posted May 21, 2019. The copyright holder for this preprint (which was not certified by peer review) is the author/funder. All rights reserved. No reuse allowed without permission.

Levetiracetam inhibits SV2A-synaptotagmin interaction

electrodes were 2.5-3.5 $\mathrm{M} \Omega$, and series resistance was compensated $70-85 \%$. Cells with series resistance $>20 \mathrm{M} \Omega$ before series resistance compensation, or leak current $>250$ pA were excluded. All experiments were performed at room temperature.

\section{Glutathione S-transferase (GST) pull-down assays}

Protein binding (pull-down) assays were performed as previously described (27). Brains from WT or BKO mice were homogenized in HBS buffer [10mM HEPES, $142 \mathrm{mM} \mathrm{NaCl}$, $2.4 \mathrm{mM} \mathrm{KCl}, 1 \mathrm{mM} \mathrm{MgCl} 2,5 \mathrm{mM}$ D-glucose, $1 \mathrm{mM}$ EGTA, 1x protease inhibitor (Roche)]. The brain homogenate was centrifuged at $1000 x g$ for 10 minutes to remove nuclei and undisrupted cells. One milligram of the resulting post nuclear supernatant was solubilized in an equal volume of solubilization buffer [20mM HEPES, 1mM EGTA, 95mM potassium acetate, $2 \%$ Triton X-100, with or without $3 \mathrm{mM} \mathrm{CaCl} 2$ and $300 \mu \mathrm{M}$ LEV or water] for two hours on ice then centrifuged at $19,000 \times$ for 30 minutes at $4^{\circ} \mathrm{C}$. The resulting supernatant (extract) was incubated with bacterially-produced GST-synaptotagmin1 (GST-Syt1) bound to Pierce ${ }^{\mathrm{TM}}$ glutathione agarose (16100) for 1 hour at $4^{\circ} \mathrm{C}$. After binding, beads were washed four times with $1 \mathrm{~mL}$ wash buffer [10mM HEPES, $1 \mathrm{mM}$ EGTA, 47.5mM potassium acetate, $1 \%$ Triton $\mathrm{X}-100$, with or without $3 \mathrm{mM} \mathrm{CaCl} 2$ and $300 \mu \mathrm{M}$ LEV or water]. Proteins were eluted with $8 \mathrm{M}$ urea dissolved in $100 \mathrm{mM}$ Tris- $\mathrm{HCl}, \mathrm{pH} 8$, or SDSPAGE sample buffer, and subjected to Western analysis.

\section{Surface Biotinylation}

Hippocampal cultures (14-16 DIV) from WT or BKO mice were treated with 300 uM LEV or water in neural media at $37^{\circ} \mathrm{C}$ for three hours. After incubation, cells were placed on ice and washed with cold phosphate buffered saline (PBS) [137mM NaCl, $2.7 \mathrm{mM} \mathrm{KCl}, 10 \mathrm{mM}$ $\left.\mathrm{NaH}_{2} \mathrm{PO}_{4}, 1.8 \mathrm{mM} \quad \mathrm{KH}_{2} \mathrm{PO}_{4}\right]$. To biotinylate surface stranded cells, cells were incubated with $2 \mathrm{mM}$ EZ-LINK Sulfo-NHS-LC-LC-Biotin (Thermo Scientific 21338) for 25 minutes at $4^{\circ} \mathrm{C}$. Cells were washed with $100 \mathrm{mM}$ glycine in
PBS for $15 \mathrm{~min}$ to quench the reaction and then washed with PBS twice. Cells were harvested and extracted in lysis buffer [10mM HEPES, $140 \mathrm{mM} \mathrm{NaCl}, 1 \%$ Triton $\mathrm{X}-100$ and protease inhibitor] for 30 minutes at $4^{\circ} \mathrm{C}$ and spun at $19,000 \times \mathrm{xg}$ for 20 minutes at $4^{\circ} \mathrm{C}$. Biotinylated proteins were isolated using Streptavidin Sepharose $®$ High Performance resin (GE 17-5113-01) and eluted with SDSPAGE sample buffer. Eluted proteins were then subjected to immunoblot analysis.

Imaging

Imaging of live cultures was performed at 14-16 DIV. Prior to imaging, neurons were incubated in neural medium with 300uM LEV or water for 3-8 hours at $37^{\circ} \mathrm{C}$. Cultures were perfused in imaging buffer $[170 \mathrm{mM} \mathrm{NaCl}$, $3.5 \mathrm{mM} \mathrm{KCl}, \quad 0.4 \mathrm{mM} \quad \mathrm{KH}_{2} \mathrm{PO}_{4}, 20 \mathrm{mM} \mathrm{N}-$ Tris(hydroxy-methyl)- methyl-2-aminoethanesulphonic acid (TES), $5 \mathrm{mM} \mathrm{NaHCO} 3,5 \mathrm{mM}$ glucose, $1.2 \mathrm{mM} \quad \mathrm{Na}_{2} \mathrm{SO}_{4}, 1.2 \mathrm{mM} \mathrm{MgCl}_{2}$, $1.3 \mathrm{mM} \mathrm{CaCl}_{2}, 10 \mu \mathrm{M} \mathrm{CNQX}$, and $50 \mu \mathrm{M}$ AP-5, $\mathrm{pH}$ 7.4] +/- 300uM LEV prior to imaging. To evoke synaptic transmission, neurons were subjected to electrical field stimulation using an RC21-BRFS chamber (Warner Instruments). A train of 200 action potentials was delivered at $40 \mathrm{~Hz}$ using a Grass SD9E Stimulator. Fluorescent images (100ms exposures) were captured using an Olympus IX-10 microscope equipped with a $60 x$ water-immersion objective and an Andor scMOS camera. Images were acquired at $2 \mathrm{~Hz}$ using Micro-Manager software and analyzed using NIH ImageJ (with Time Series Analyzer V3 plugin). Responding boutons were identified and total fluorescence intensity monitored over time. Fluorescence intensity was corrected for background (47). Endocytic time constants were determined by fitting the mono-exponential decay of fluorescence intensity using Prism (Graphpad) software.

\section{Statistical Methods}

All statistical analyses were performed using Prism (Graphpad) software. Data analysis was performed with the assistance from the Department of Biostatistics Consulting Service at the University of Washington. 
bioRxiv preprint doi: https://doi.org/10.1101/640185; this version posted May 21, 2019. The copyright holder for this preprint (which was not certified by peer review) is the author/funder. All rights reserved. No reuse allowed without permission.

Levetiracetam inhibits SV2A-synaptotagmin interaction

\section{Acknowledgements}

This work was supported, in part, by grants from the National Institutes of Mental Health (R21MH099741 to SB), National Institutes of General Medical Sciences (T32GM007270 and T32GM007750 to KC).

\section{Literature Cited}

1. Farooq, M. U., Bhatt, A., Majid, A., Gupta, R., Khasnis, A., and Kassab, M. Y. (2009) Levetiracetam for managing neurologic and psychiatric disorders. American journal of health-system pharmacy : AJHP : official journal of the American Society of HealthSystem Pharmacists 66, 541-561

2. Loscher, W., Gillard, M., Sands, Z. A., Kaminski, R. M., and Klitgaard, H. (2016) Synaptic Vesicle Glycoprotein 2A Ligands in the Treatment of Epilepsy and Beyond. CNS drugs 30, 1055-1077

3. Mendoza-Torreblanca, J. G., Vanoye-Carlo, A., Phillips-Farfan, B. V., Carmona-Aparicio, L., and Gomez-Lira, G. (2013) Synaptic vesicle protein 2A: basic facts and role in synaptic function. Eur J Neurosci 38, 3529-3539

4. Lynch, B. A., Lambeng, N., Nocka, K., Kensel-Hammes, P., Bajjalieh, S. M., Matagne, A., and Fuks, B. (2004) The synaptic vesicle protein SV2A is the binding site for the antiepileptic drug levetiracetam. Proc Natl Acad Sci U S A 101, 9861-9866

5. Kaminski, R. M., Gillard, M., Leclercq, K., Hanon, E., Lorent, G., Dassesse, D., Matagne, A., and Klitgaard, H. (2009) Proepileptic phenotype of SV2A-deficient mice is associated with reduced anticonvulsant efficacy of levetiracetam. Epilepsia 50, 17291740

6. Kaminski, R. M., Gillard, M., and Klitgaard, H. (2012) Targeting SV2A for Discovery of Antiepileptic Drugs. in Jasper's Basic Mechanisms of the Epilepsies (Noebels, J. L., Avoli, M., Rogawski, M. A., Olsen, R. W., and Delgado-Escueta, A. V. eds.), 4th Ed., Bethesda (MD). pp

\section{Disclosure}

The authors have no conflict of interest to disclose. We confirm that we have read the Journal's position on issues involved in ethical publication and affirm that this report is consistent with those guidelines.

7. Nowack, A., Yao, J., Custer, K. L., and Bajjalieh, S. M. (2010) SV2 regulates neurotransmitter release via multiple mechanisms. American journal of physiology. Cell physiology 299, C960-967

8. Madeo, M., Kovacs, A. D., and Pearce, D. A. (2014) The human synaptic vesicle protein, SV2A, functions as a galactose transporter in Saccharomyces cerevisiae. J Biol Chem 289, 33066-33071

9. Yao, J., Nowack, A., Kensel-Hammes, P., Gardner, R. G., and Bajjalieh, S. M. (2010) Cotrafficking of SV2 and synaptotagmin at the synapse. J Neurosci 30, 5569-5578

10. Zhang, N., Gordon, S. L., Fritsch, M. J., Esoof, N., Campbell, D. G., Gourlay, R., Velupillai, S., Macartney, T., Peggie, M., van Aalten, D. M., Cousin, M. A., and Alessi, D. R. (2015) Phosphorylation of synaptic vesicle protein $2 \mathrm{~A}$ at Thr84 by casein kinase 1 family kinases controls the specific retrieval of synaptotagmin-1. J Neurosci 35, 2492-2507

11. Kaempf, N., Kochlamazashvili, G., Puchkov, D., Maritzen, T., Bajjalieh, S. M., Kononenko, N. L., and Haucke, V. (2015) Overlapping functions of stonin 2 and SV2 in sorting of the calcium sensor synaptotagmin 1 to synaptic vesicles. Proc Natl Acad Sci U S A 112, 7297-7302

12. Gronborg, M., Pavlos, N. J., Brunk, I., Chua, J. J., Munster-Wandowski, A., Riedel, D., Ahnert-Hilger, G., Urlaub, H., and Jahn, R. (2010) Quantitative comparison of glutamatergic and GABAergic synaptic vesicles unveils selectivity for few proteins including MAL2, a novel synaptic vesicle protein. J Neurosci 30, 2-12

13. Bajjalieh, S. M., Frantz, G. D., Weimann, J. M., McConnell, S. K., and Scheller, R. H. (1994) Differential expression of synaptic 
bioRxiv preprint doi: https://doi.org/10.1101/640185; this version posted May 21, 2019. The copyright holder for this preprint (which was not certified by peer review) is the author/funder. All rights reserved. No reuse allowed without permission.

Levetiracetam inhibits SV2A-synaptotagmin interaction

vesicle protein 2 (SV2) isoforms. J Neurosci 14, 5223-5235

14. Crowder, K. M., Gunther, J. M., Jones, T. A., Hale, B. D., Zhang, H. Z., Peterson, M. R., Scheller, R. H., Chavkin, C., and Bajjalieh, S. M. (1999) Abnormal neurotransmission in mice lacking synaptic vesicle protein $2 \mathrm{~A}$ (SV2A). Proc Natl Acad Sci U S A 96, 15268-15273

15. Janz, R., Goda, Y., Geppert, M., Missler, M., and Sudhof, T. C. (1999) SV2A and SV2B function as redundant $\mathrm{Ca} 2+$ regulators in neurotransmitter release. Neuron 24, 10031016

16. Matveeva, E. A., Vanaman, T. C., Whiteheart, S. W., and Slevin, J. T. (2007) Asymmetric accumulation of hippocampal 7S SNARE complexes occurs regardless of kindling paradigm. Epilepsy Res 73, 266-274

17. Matveeva, E. A., Vanaman, T. C., Whiteheart, S. W., and Slevin, J. T. (2008) Levetiracetam prevents kindling-induced asymmetric accumulation of hippocampal 7S SNARE complexes. Epilepsia 49, 1749-1758

18. Ohno, Y., Ishihara, S., Terada, R., Kikuta, M., Sofue, N., Kawai, Y., Serikawa, T., and Sasa, M. (2009) Preferential increase in the hippocampal synaptic vesicle protein $2 \mathrm{~A}$ (SV2A) by pentylenetetrazole kindling. Biochem Biophys Res Commun 390, 415-420

19. Nowack, A., Malarkey, E. B., Yao, J., Bleckert, A., Hill, J., and Bajjalieh, S. M. (2011) Levetiracetam reverses synaptic deficits produced by overexpression of SV2A. PLOS One 6, e29560

20. Meehan, A. L., Yang, X., Yuan, L. L., and Rothman, S. M. (2012) Levetiracetam has an activity-dependent effect on inhibitory transmission. Epilepsia 53, 469-476

21. Meehan, A. L., Yang, X., McAdams, B. D., Yuan, L., and Rothman, S. M. (2011) A New Mechanism for Antiepileptic Drug Action: Vesicular Entry May Mediate the Effects of Levetiracetam. J Neurophysiol 106, 1227-1239

22. Dobrunz, L. E., Huang, E. P., and Stevens, C. F. (1997) Very short-term plasticity in hippocampal synapses. Proc Natl Acad Sci U S A 94, 14843-14847
23. Yang, X. F., Weisenfeld, A., and Rothman, S. M. (2007) Prolonged Exposure to Levetiracetam Reveals a Presynaptic Effect on Neurotransmission. Epilepsia 48, 1861-1869

24. Zucker, R. S., and Regehr, W. G. (2002) Short-term synaptic plasticity. Annual review of physiology 64, 355-405

25. Custer, K. L., Austin, N. S., Sullivan, J. M., and Bajjalieh, S. M. (2006) Synaptic vesicle protein 2 enhances release probability at quiescent synapses. J Neurosci 26, 1303-1313

26. Schivell, A. E., Mochida, S., KenselHammes, P., Custer, K. L., and Bajjalieh, S. M. (2005) SV2A and SV2C contain a unique synaptotagmin-binding site. Mol Cell Neurosci 29, 56-64

27. Schivell, A. E., Batchelor, R. H., and Bajjalieh, S. M. (1996) Isoform-specific, calcium-regulated interaction of the synaptic vesicle proteins SV2 and synaptotagmin. The Journal of biological chemistry 271, 2777027775

28. Pyle, R. A., Schivell, A. E., Hidaka, H., and Bajjalieh, S. M. (2000) Phosphorylation of synaptic vesicle protein 2 modulates binding to synaptotagmin. The Journal of biological chemistry 275, 17195-17200

29. Lazzell, D. R., Belizaire, R., Thakur, P., Sherry, D. M., and Janz, R. (2004) SV2B regulates synaptotagmin 1 by direct interaction. J Biol Chem 279, 52124-52131

30. Stevens, C. F., and Sullivan, J. M. (2003) The synaptotagmin C2A domain is part of the calcium sensor controlling fast synaptic transmission. Neuron 39, 299-308

31. Striegel, A. R., Biela, L. M., Evans, C. S., Wang, Z., Delehoy, J. B., Sutton, R. B., Chapman, E. R., and Reist, N. E. (2012) Calcium binding by synaptotagmin's C2A domain is an essential element of the electrostatic switch that triggers synchronous synaptic transmission. The Journal of neuroscience : the official journal of the Society for Neuroscience 32, 1253-1260

32. Brunger, A. T., Choi, U. B., Lai, Y., Leitz, J., and Zhou, Q. (2018) Molecular Mechanisms of Fast Neurotransmitter Release. Annual review of biophysics 47, 469-497 
bioRxiv preprint doi: https://doi.org/10.1101/640185; this version posted May 21, 2019. The copyright holder for this preprint (which was not certified by peer review) is the author/funder. All rights reserved. No reuse allowed without permission.

Levetiracetam inhibits SV2A-synaptotagmin interaction

33. Yao, J., Kwon, S. E., Gaffaney, J. D., Dunning, F. M., and Chapman, E. R. (2011) Uncoupling the roles of synaptotagmin I during endo- and exocytosis of synaptic vesicles. Nature neuroscience 15, 243-249

34. Yao, L. H., Rao, Y., Varga, K., Wang, C. Y., Xiao, P., Lindau, M., and Gong, L. W. (2012) Synaptotagmin 1 is necessary for the $\mathrm{Ca} 2+$ dependence of clathrin-mediated endocytosis. The Journal of neuroscience : the official journal of the Society for Neuroscience 32, 3778-3785

35. Haucke, V., and De Camilli, P. (1999) AP2 recruitment to synaptotagmin stimulated by tyrosine-based endocytic motifs. Science 285, 1268-1271

36. Miesenbock, G., De Angelis, D. A., and Rothman, J. E. (1998) Visualizing secretion and synaptic transmission with $\mathrm{pH}$-sensitive green fluorescent proteins. Nature 394, 192195

37. Krook-Magnuson, E., Armstrong, C., Bui, A., Lew, S., Oijala, M., and Soltesz, I. (2015) In vivo evaluation of the dentate gate theory in epilepsy. J Physiol 593, 2379-2388

38. Bernard, C. (2012) Alterations in synaptic function in epilepsy. in Jasper's Basic Mechanisms of the Epilepsies (th, Noebels, J. L., Avoli, M., Rogawski, M. A., Olsen, R. W., and Delgado-Escueta, A. V. eds.), Bethesda (MD). pp

39. Ohno, Y., Okumura, T., Terada, R., Ishihara, S., Serikawa, T., and Sasa, M. Kindling-associated SV2A expression in hilar GABAergic interneurons of the mouse dentate gyrus. Neurosci Lett 510, 93-98

40. Feany, M. B., Lee, S., Edwards, R. H., and Buckley, K. M. (1992) The synaptic vesicle protein SV2 is a novel type of transmembrane transporter. Cell 70, 861-867

41. Janz, R., Goda, Y., Geppert, M., Missler, M., and Sudhof, T. C. (1999) SV2A and SV2B function as redundant $\mathrm{Ca} 2+$ regulators in neurotransmitter release. Neuron 24, 10031016

42. Wan, Q.-F., Zhou, Z.-Y., Thakur, P., Vila, A., Sherry, D. M., Janz, R., and Heidelberger, R. (2010) SV2 acts via presynaptic calcium to regulate neurotransmitter release. Neuron 66, 884-895

43. Schivell, A. E., Batchelor, R. H., and Bajjalieh, S. M. (1996) Isoform-specific, calcium-regulated interaction of the synaptic vesicle proteins SV2 and synaptotagmin. J. Biol. Chem. 271, 27770-27775

44. Gordon, S. L., and Cousin, M. A. (2016) The iTRAPs: Guardians of Synaptic Vesicle Cargo Retrieval During Endocytosis. Frontiers in synaptic neuroscience 8, 1

45. Xu, T., and Bajjalieh, S. M. (2001) SV2 modulates the size of the readily releasable pool of secretory vesicles. Nat Cell Biol 3, 691698

46. Garcia-Perez, E., Mahfooz, K., Covita, J., Zandueta, A., and Wesseling, J. F. (2015) Levetiracetam accelerates the onset of supply rate depression in synaptic vesicle trafficking. Epilepsia 56, 535-545

47. Nicholson-Fish, J. C., Smillie, K. J., and Cousin, M. A. (2016) Monitoring activitydependent bulk endocytosis with the genetically-encoded reporter VAMP4-pHluorin. Journal of neuroscience methods 266, 1-10 